\title{
CYLINDRICAL WAVES IN THE DIELECTRIC SLAB
}

\author{
V. Ivaška and V. Jonkus \\ Department of Radiophysics, Faculty of Physics, Vilnius University, Saulètekio 9, LT-10222 Vilnius, Lithuania \\ E-mail: vytautas.jonkus@ff.vu.lt
}

Received 17 September 2007; revised 26 January 2007; accepted 22 February 2008

\begin{abstract}
In this paper a theoretical approach to electromagnetic field distribution in a dielectric slab is discussed. Electrodynamical formulation of the problem involves Hertzian potentials. The electromagnetic field distribution in the slab depends on a function $U(x, y)$ that obeys the two-dimensional Helmholtz equation. Cylindrical waves are one of possible electromagnetic field configurations in the slab that occurs when the function $U(x, y)$ is expressed in cylindrical coordinates.
\end{abstract}

Keywords: electromagnetic field modes, dielectric slab

PACS: 47.65.-d, 03.50.De

\section{Introduction}

Dielectric waveguides and dielectric resonators are analysed in many academic books and journals. In most cases the plane wave is supposed to propagate in dielectric media [1-3] Then, electromagnetic field modes and dispersion relations follow as a consequence of boundary condition restrictions. In this paper the theoretical approach involving Hertzian potentials is used. This more complicated mathematical approach to the problem leads to cylindrical configuration waves. Cylindrical configuration waves are supposed to be a solution of the wave equation, having a form

$$
\begin{aligned}
& \varphi(x, y, z, t)=U(x, y) \mathrm{e}^{\mathrm{i} \omega t} \mathrm{e}^{ \pm \mathrm{i} h z}, \text { inside } \\
& \varphi(x, y, z, t)=U(x, y) \mathrm{e}^{\mathrm{i} \omega t} \mathrm{e}^{-h|z|}, \text { outside }
\end{aligned}
$$

where $z$ is the symmetry axis; constant $h$ must be determined from the corresponding dispersion relation.

The conventional way to calculate characteristics related to a dielectric slab is to use the plane wave model. Even such complicated problem as Bessel beam propagation and reflection in a dielectric slab can be solved in terms of plane waves [2]. In our notation the plane wave corresponds to $U(x, y)=\exp \left[\mathrm{i}\left( \pm h_{x} x \pm h_{y} y\right)\right]$ in Eq. (1).

In this paper standing and propagating waves in the dielectric slab are derived on the basis of Hertzian potentials. These potentials have a fixed direction (perpendicular to the surface of the slab) and obey the wave equation. The solution has the form of Eq. (1) where
$U(x, y)$ is required to satisfy an appropriate equation that will be discussed in the next section. There are two types of Hertzian potentials: electric $(e)$ and magnetic $(\mathrm{m})$; therefore, the electromagnetic field configuration has transverse magnetic or transverse electric field (see schematic plot in Fig. 1). Electromagnetic field classification in $T M$ and $T E$ modes is dicussed in Subsection 2.4. Hertzian potentials are independent of the coordinate system in which the function $U(x, y)$ is expressed. Therefore, numerical results and graphical illustration use the cylindrical coordinate $\{\rho, \alpha, z\}$ system where the $z$ axis corresponds to the symmetry axis in Eq. (1) and $U(x, y)=U(\rho, \alpha)$.

The dielectric slab is supposed to have infinite dimensions in $x$ and $y$ directions of the Cartesian coordinate system, while in the $z$ direction it has thickness

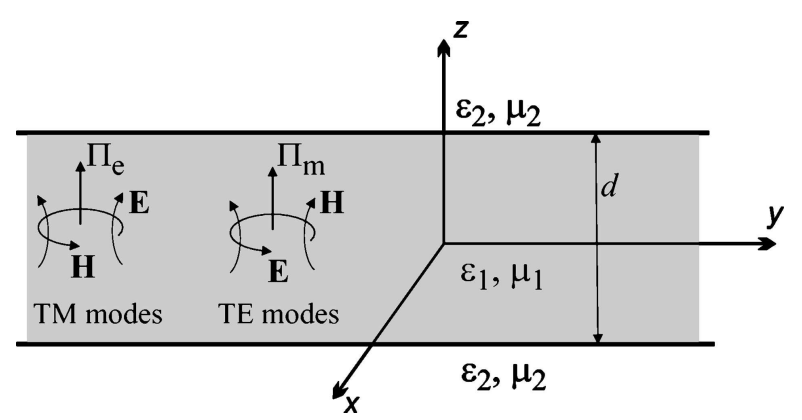

Fig. 1. Dielectric slab of finite thickness $d$ along the $z$ axis, of a medium with permittivity $\varepsilon_{1}$ and permeability $\mu_{1}$, surrounded on both sides by a different medium with permittivity $\varepsilon_{2}$ and permeability $\mu_{2}$. 
$d$ (see Fig. 1). The dielectric is supposed to be lossless media characterized by permittivity and permeability.

\section{Hertzian potentials}

Magnetic and electric field distributions are derived from Hertzian potentials (electric and / or magnetic) assuming that the potentials have only one perpendicular component [4]. Hertzian potentials are expressed as follows:

$$
\begin{aligned}
& e, m=\mathbf{z}_{0} A_{1}^{ \pm} U(x, y) \mathrm{e}^{ \pm \mathrm{i} h_{1} z} \mathrm{e}^{\mathrm{i} \omega t},|z| \leq d / 2, \\
& e, m=\mathbf{z}_{0} A_{2}^{ \pm} U(x, y) \mathrm{e}^{-h_{2}|z|} \mathrm{e}^{\mathrm{i} \omega t},|z| \geq d / 2,
\end{aligned}
$$

where Eqs. (2a) and (2b) expressions are valid inside $(|z| \leq d / 2)$ and outside $(|z| \geq d / 2)$ the dielectric slab, respectively; $\mathbf{z}_{0}$ is the unit vector of the Cartesian coordinate system; $A_{1}^{ \pm}$and $A_{2}^{ \pm}$are amplitudes that differ for $z>0$ and $z<0$. As the Eq. (2b) describes the electromagnetic field outside the slab, then it is required that the electromagnetic field would disappear in the infinity along the direction perpendicular to the slab surface. Therefore, the sign of the exponent power in Eq. (2b) is negative while the corresponding sign in the Eq. (2a) can be either positive or negative.

For convenience we rearrange Eq. (2a) as follows: we take the sum or the difference of Eq. (2a) with different power signs of the exponents. Consequenly, the rearranged expression contain $\sin \left(h_{1} z\right), \cos \left(h_{1} z\right)$, and Hertzian potential can be symmetrical or asymmetrical along the perpendicular axis to the slab.

The function $U(x, y)$ is required to satisfy the following equation:

$$
\Delta_{\perp} U+\beta^{2} U=0 .
$$

Part of Laplace operator $\Delta_{\perp}$ does not include derivative with respect to coordinate $z$. Equation (3) is obtained from the requirement for Hertzian potentials to satisfy the wave equation [4]. This requirement applied to both (2) expressions leads to additional relations among $h_{1}$, $h_{2}$, and $\beta$ :

$$
\omega^{2} \varepsilon_{1} \mu_{1}-h_{1}^{2}=\omega^{2} \varepsilon_{2} \mu_{2}+h_{2}^{2}=\beta^{2} .
$$

As a result, the quantity $\beta$ is independent of the function $U(x, y)$.

Two cases are distinguished depending on whether $e$ or $m$ is zero: (i) magnetic field is transversal $\left(H_{z}=0\right)$ to the symmetry axis when $m=0-$ this is the $T M$ configuration; (ii) electric field is transversal $\left(E_{z}=0\right)$ to the symmetry axis when $e=0-$ this is the $T E$

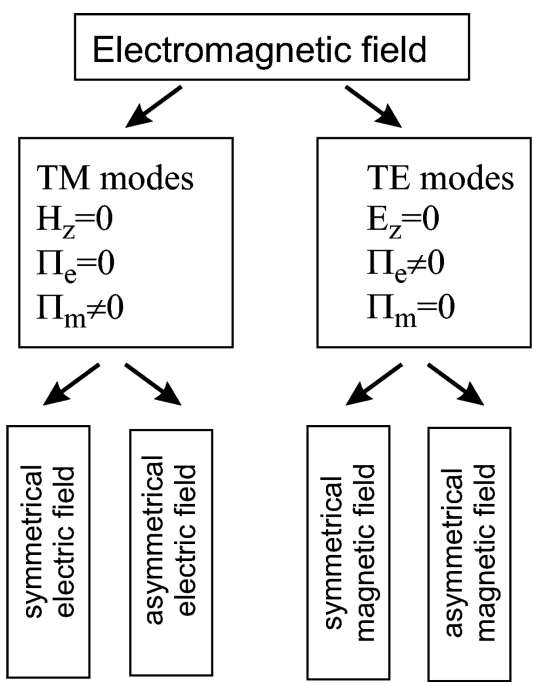

Fig. 2. Solution classification.

configuration. The electromagnetic field classification scheme is shown in Fig. 2.

The object of further caculations is the electromagnetic field having cylindrical symmetry; therefore, the cylindrical coordinate system $\rho, \alpha, z$ is used.

\subsection{Electromagnetic field in the case $m=0$}

For the ${ }_{m}=0$ case electric and magnetic field expressions outside the slab are as follows:

$$
\begin{aligned}
& \mathbf{E}=A_{2}^{ \pm} \frac{1}{\varepsilon_{2}}\left(\mp h_{2} \mathbf{L}+\beta^{2} U \mathbf{z}_{0}\right) \mathrm{e}^{-h_{2}|z|} \mathrm{e}^{\mathrm{i} \omega t}, \\
& \mathbf{H}=-\mathrm{i} \omega A_{2}^{ \pm} \mathbf{S} \mathrm{e}^{-h_{2}|z|} \mathrm{e}^{\mathrm{i} \omega t} .
\end{aligned}
$$

The upper sign at $A_{2}^{ \pm}$constant means that $A_{2}^{ \pm}$is different for $z>d / 2$ and $z<-d / 2$. Newly introduced vectors $\mathbf{L}$ and $\mathbf{S}$ in the Cartesian and cylindrical coordinate systems are

$$
\begin{aligned}
& \mathbf{L}=\frac{\partial U}{\partial x} \mathbf{x}_{0}+\frac{\partial U}{\partial y} \mathbf{y}_{0}=\frac{\partial U}{\partial \rho} \boldsymbol{\rho}_{0}+\frac{1}{\rho} \frac{\partial U}{\partial \alpha} \boldsymbol{\alpha}_{0}, \\
& \mathbf{S}=\frac{\partial U}{\partial x} \mathbf{x}_{0}-\frac{\partial U}{\partial y} \mathbf{y}_{0}=-\frac{1}{\rho} \frac{\partial U}{\partial \alpha} \boldsymbol{\rho}_{0}+\frac{\partial U}{\partial \rho} \boldsymbol{\alpha}_{0},
\end{aligned}
$$

where $\mathbf{x}_{0}, \mathbf{y}_{0}$ and $\boldsymbol{\alpha}_{0}, \boldsymbol{\rho}_{0}$ are unity vectors of the Cartesian and the cylindrical coordinate system, respectively. Magnetic field inside the slab has two configurations: 
symmetrical and asymmetrical. For the symmetrical magnetic field we have

$$
\mathbf{E}=A_{1} \frac{2}{\varepsilon_{1}}\left\{-h_{1} \mathbf{L} \sin \left(h_{1} z\right)+\beta^{2} U \cos \left(h_{1} z\right) \mathbf{z}_{0}\right\} \mathrm{e}^{\mathrm{i} \omega t},
$$

$$
\mathbf{H}=-2 \mathrm{i} \omega A_{1} \mathbf{S} \cos \left(h_{1} z\right) \mathrm{e}^{\mathrm{i} \omega t},
$$

and for asymmetrical magnetic field

$$
\mathbf{E}=A_{1} \frac{2 \mathrm{i}}{\varepsilon_{1}}\left\{h_{1} \mathbf{L} \cos \left(h_{1} z\right)+\beta^{2} U \sin \left(h_{1} z\right) \mathbf{z}_{0}\right\} \mathrm{e}^{\mathrm{i} \omega t},
$$

$$
\mathbf{H}=2 \omega A_{1} \mathbf{S} \sin \left(h_{1} z\right) \mathrm{e}^{\mathrm{i} \omega t} .
$$

Boundary conditions for tangential components of vectors $\mathbf{E}$ and $\mathbf{H}$ determine relation among $A_{1}, A_{2}^{ \pm}, \beta$, and $\omega$. In the case of symmetrical magnetic field we have

$$
\begin{aligned}
\frac{\varepsilon_{1} \sqrt{\beta^{2}-\omega^{2} \varepsilon_{2} \mu_{2}}}{\varepsilon_{2} \sqrt{\omega^{2} \varepsilon_{1} \mu_{1}-\beta^{2}}} & =\tan \left(\frac{d}{2} \sqrt{\omega^{2} \varepsilon_{1} \mu_{1}-\beta^{2}}\right), \\
\frac{A_{2}^{ \pm}}{A_{1}} & =2 \cos \left(\frac{d}{2} \sqrt{\omega^{2} \varepsilon_{1} \mu_{1}-\beta^{2}}\right) \\
& \times \exp \left(\frac{d}{2} \sqrt{\beta^{2}-\omega^{2} \varepsilon_{2} \mu_{2}}\right),
\end{aligned}
$$

and in the case of asymmetrical magnetic field we have

$$
\begin{aligned}
& \frac{\varepsilon_{2} \sqrt{\omega^{2} \varepsilon_{1} \mu_{1}-\beta^{2}}}{\varepsilon_{1} \sqrt{\beta^{2}-\omega^{2} \varepsilon_{2} \mu_{2}}}=-\tan \left(\frac{d}{2} \sqrt{\omega^{2} \varepsilon_{1} \mu_{1}-\beta^{2}}\right), \\
& \frac{A_{2}^{ \pm}}{A_{1}}= \pm 2 \operatorname{iin}\left(\frac{d}{2} \sqrt{\omega^{2} \varepsilon_{1} \mu_{1}-\beta^{2}}\right) \\
& \times \exp \left(\frac{d}{2} \sqrt{\beta^{2}-\omega^{2} \varepsilon_{2} \mu_{2}}\right) .
\end{aligned}
$$

As follows from Eqs. (7) and (8), the magnetic field has no $H_{z}$ component. These are $T M_{n}$ modes, where index $n$ denotes the root number of Eq. (9a) or (10a), because these transcendental equations have to be solved with respect to $\beta$ and can have more than one root. The solution of Eq. (3) in cylindrical coordinate system can be found in [4], where solution is expressed in terms of Bessel and trigonometric functions.

\subsection{Electromagnetic field in the case $e_{e}=0$}

Electric and magnetic field outside the slab are as follows:

$$
\begin{aligned}
& \mathbf{E}=A_{2}^{ \pm} \frac{\mathrm{i} \omega}{\varepsilon_{2}} \mathbf{S} \mathrm{e}^{-h_{2}|z|} \mathrm{e}^{\mathrm{i} \omega t}, \\
& \mathbf{H}=A_{2}^{ \pm} \frac{1}{\varepsilon_{2} \mu_{2}}\left(\mp h_{2} \mathbf{L}+\beta U \mathbf{z}_{0}\right) \mathrm{e}^{-h_{2}|z|} \mathrm{e}^{\mathrm{i} \omega t} .
\end{aligned}
$$

In case $e_{e}=0$, the electric field inside the slab has two configurations: symmetrical and asymmetrical. Electromagnetic field inside the slab for the symmetrical electric field case is expressed as follows:

$$
\begin{aligned}
\mathbf{E}=A_{1} \frac{2 \mathrm{i} \omega}{\varepsilon_{1}} \mathbf{S} & \cos \left(h_{1} z\right) \mathrm{e}^{\mathrm{i} \omega t}, \\
\mathbf{H}=A_{1} \frac{2}{\varepsilon_{1} \mu_{1}} & {\left[-h_{1} \mathbf{L} \sin \left(h_{1} z\right)\right.} \\
& \left.+\beta^{2} U \cos \left(h_{1} z\right) \mathbf{z}_{0}\right] \mathrm{e}^{\mathrm{i} \omega t} .
\end{aligned}
$$

Electromagnetic field expressions inside the slab for the asymmetrical electric field case are

$$
\begin{aligned}
\mathbf{E}=-A_{1} \frac{2 \omega}{\varepsilon_{1}} & \mathbf{S} \sin \left(h_{1} z\right) \mathrm{e}^{i \omega t}, \\
\mathbf{H}=A_{1} \frac{2 \mathrm{i}}{\varepsilon_{1} \mu_{1}}[ & h_{1} \mathbf{L} \cos \left(h_{1} z\right) \\
& \left.+\beta^{2} U \sin \left(h_{1} z\right) \mathbf{z}_{0}\right] \mathrm{e}^{\mathrm{i} \omega t} .
\end{aligned}
$$

Boundary conditions in the symmetrical electric field case lead to relations

$$
\begin{aligned}
& \frac{\mu_{1} \sqrt{\beta^{2}-\omega^{2} \varepsilon_{2} \mu_{2}}}{\mu_{2} \sqrt{\omega^{2} \varepsilon_{1} \mu_{1}-\beta^{2}}}=\tan \left(\frac{d}{2} \sqrt{\omega^{2} \varepsilon_{1} \mu_{1}-\beta^{2}}\right), \\
& \frac{A_{2}^{ \pm}}{A_{1}}=2 \frac{\varepsilon_{2}}{\varepsilon_{1}} \cos \left(\frac{d}{2} \sqrt{\omega^{2} \varepsilon_{1} \mu_{1}-\beta^{2}}\right) \\
& \\
& \times \exp \left(\frac{d}{2} \sqrt{\beta^{2}-\omega^{2} \varepsilon_{2} \mu_{2}}\right),
\end{aligned}
$$


and in the asymmetrical electric field case they are as follows:

$$
\begin{aligned}
& \frac{\mu_{2} \sqrt{\omega^{2} \varepsilon_{1} \mu_{1}-\beta^{2}}}{\mu_{1} \sqrt{\beta^{2}-\omega^{2} \varepsilon_{2} \mu_{2}}}=-\tan \left(\frac{d}{2} \sqrt{\omega^{2} \varepsilon_{1} \mu_{1}-\beta^{2}}\right), \\
& \frac{A_{2}^{ \pm}}{A_{1}}= \pm 2 \mathrm{i} \frac{\varepsilon_{2}}{\varepsilon_{1}} \sin \left(\frac{d}{2} \sqrt{\omega^{2} \varepsilon_{1} \mu_{1}-\beta^{2}}\right) \\
& \times \exp \left(\frac{d}{2} \sqrt{\beta^{2}-\omega^{2} \varepsilon_{2} \mu_{2}}\right) .
\end{aligned}
$$

As follows from Eqs. (12) and (13), the electric field has no $E_{z}$ component. This electromagnetic field configuration is called $T E_{n}$ modes, where index $n$ denotes the root number of Eq. (14a) or (15a).

\subsection{Travelling waves}

Travelling wave with cylindrical configuration follows as a special case with function $U$. Suppose, the function $U(\rho, \alpha)$ is expressed in the cylindrical coordinate system. The electromagnetic field will have the form of a travelling wave when $U(\rho, \alpha) \sim \mathrm{e}^{-\mathrm{i} \beta \rho}$. The solution for the travelling wave of Eq. (3) that is independent of the angle $\alpha$ will be [5]

$$
U(\rho, \alpha)=c_{1} \mathrm{H}_{0}^{(2)}(\beta \rho),
$$

where $\mathrm{H}_{0}^{(2)}(x)$ is Hankel function of the second kind. The function $U(\rho, \alpha)$, expressed in this way, far from the symmetry axis becomes

$$
\lim _{\rho \rightarrow \infty} U(\rho, \alpha) \sim \mathrm{e}^{-\mathrm{i} \beta \rho} .
$$

Here asymptotic expansion of function $\mathrm{H}_{0}^{(2)}$ is used for $\rho \rightarrow \infty$ [5]. The electric field outside the slab for the case $\Pi_{m}=0$ is obtained from Eq. (5) in respect of Eq. (16) to be

$$
\lim _{\rho \rightarrow \infty} \mathbf{E} \sim \pm \mathrm{i} h_{2} \mathrm{e}^{\mathrm{i}(\omega t-\beta \rho)} \boldsymbol{\rho}_{0}+\beta \mathrm{e}^{\mathrm{i}(\omega t-\beta \rho)} \mathbf{z}_{0} .
$$

This indicates that the electromagnetic field far from the symmetry axis has the same form as the travelling wave. Moreover, the electric field is elliptically polarized and rotates to an opposite direction at the upper space $z>$ $d / 2$ of the slab as compared to the lower one $z<-d / 2$, because plus and minus signs in Eq. (18) correspond to $z>d / 2$ and $z<-d / 2$, respectively.

The case $\Pi_{e}=0$ leads to similar results.

\subsection{Poynting vector and electromagnetic field modes}

The time-average Poynting vector is defined as follows:

$$
\mathbf{P}=\frac{1}{2}[\mathbf{E} \times \stackrel{*}{\mathbf{H}}] .
$$

In the case when $\Pi_{m}=0$, the electric and the magnetic fields in Cartesian coordinate system can be written in the following form:

$$
\begin{aligned}
& \mathbf{E}=\mathbf{x}_{0} E_{x}+\mathbf{y}_{0} E_{y}+\mathbf{z}_{0} E_{z}, \\
& \mathbf{H}=\mathbf{x}_{0} H_{x}+\mathbf{y}_{0} H_{y} .
\end{aligned}
$$

Then the Poynting vector is expressed as follows:

$$
\mathbf{P}=-\mathbf{x}_{0} E_{z} \stackrel{*}{H}_{y}+\mathbf{y}_{0} E_{z} \stackrel{*}{H}_{x}+\mathbf{z}_{0}\left(E_{x} \stackrel{*}{H}_{y}-E_{y} \stackrel{*}{H}_{x}\right) .
$$

The Poynting vector component $P_{z}$ is a pure imaginary function. This indicates that there is no power flow along the $z$ direction. Whether $P_{x}$ and $P_{y}$ components have the real parts, depends on function $U(x, y)$ in Eq. (6). If $U(x, y)$ is a pure real function, then the Poynting vector is pure imaginary function. This happens to be a condition for standing waves. If $U(x, y)$ has an imaginary part, then Poynting vector has a real part:

$$
\operatorname{Re} \mathbf{P}=\frac{1}{2}\left[-\mathbf{x}_{0} \operatorname{Re}\left(E_{z} \stackrel{*}{H}_{y}\right)+\mathbf{y}_{0} \operatorname{Re}\left(E_{z} \stackrel{*}{H}_{x}\right)\right] .
$$

In this case the power flow direction is parallel to the surface of the slab. Moreover, the real part $\operatorname{Re}\left[\mathbf{E} \times \mathbf{H}^{*}\right] / 2$ of the Poynting vector is perpendicular to the $\mathbf{H}$ vector, but it is not perpendicular to the $\mathbf{E}$ vector. The electromagnetic field configuration when magnetic field is perpendicular to the power flow direction is called $T M$ mode.

Similarly, when $\Pi_{e}=0$, the power flow analysis leads to these conclusions: standing waves or travelling waves take place when $\operatorname{Im} U(x, y)=0$ or $\operatorname{Im} U(x, y) \neq$ 0 , respectively. The electric field is perpendicular to $\operatorname{Re}\left[\mathbf{E} \times \stackrel{H}{*}^{*}\right] / 2$, consequently, the case $\Pi_{e}=0$ corresponds to $T E$ modes.

\subsection{Numerical results and conclusions}

Graphical illustrations of obtained expressions are plotted in Figs. 3, 4, and 5. These figures illustrate the case with $T M_{1}$ mode, when $\beta$ is the lowest root of Eq. (9) and the function $U(\rho, \alpha)$ is chosen to be $U(\rho, \alpha)=C \mathbf{J}_{0}(\beta \rho)$, where $\mathbf{J}_{0}(x)$ is the Bessel function. 


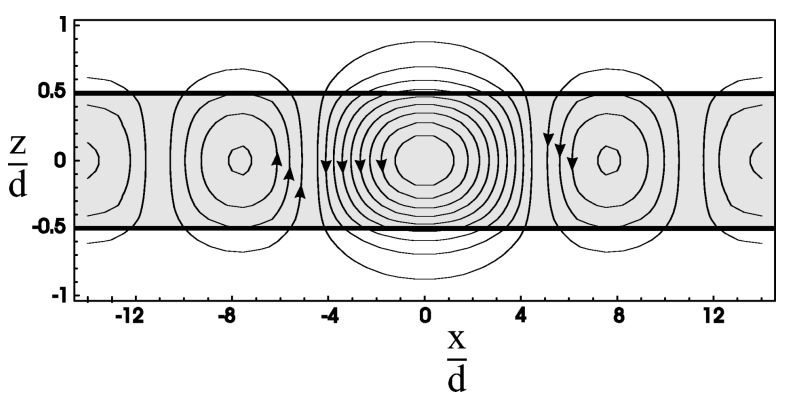

Fig. 3. Magnetic field lines. $T E_{1}$ mode, $\varepsilon_{1}=10, \varepsilon_{2}=1, f=$ $1 \mathrm{GHz}$, symmetrical electric field case.

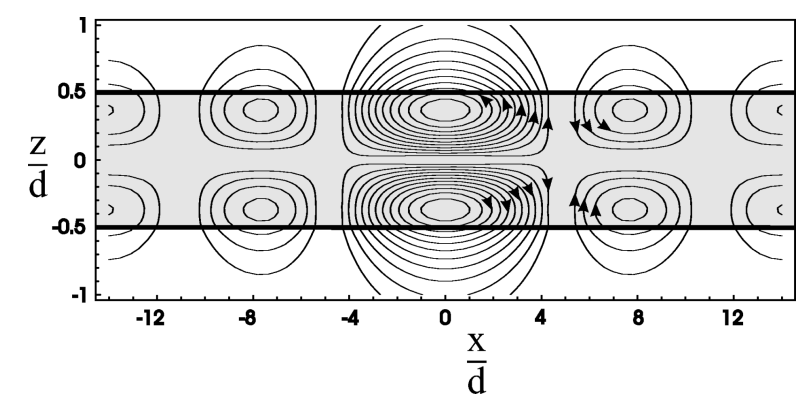

Fig. 4. Magnetic field lines. $T E_{1}$ mode, $\varepsilon_{1}=10, \varepsilon_{2}=1, f=$ $1 \mathrm{GHz}$, asymmetrical electric field case.

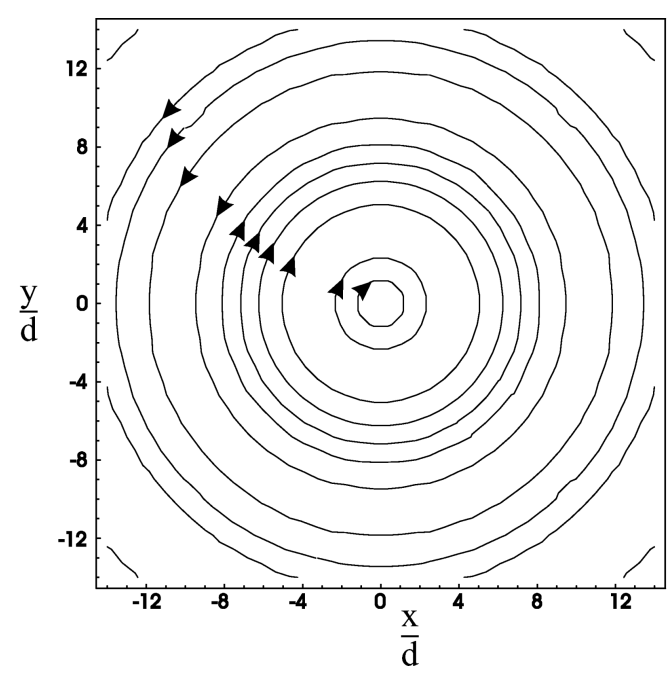

The distribution of electromagnetic field in the dielectric slab is examined with the Hertzian vector formalism. The obtained dispersion relations (9a), (10a), (14a), (15a) are independent of function $U(x, y)$. It implies that there are an infinite number of electromagnetic field configurations in the dielectric slab with the same dispersion characteristics. Exact electromagnetic field configuration in dielectric media depends on exciting conditions of the electromagnetic field. The obtained dispersion relations can be reduced to wellknown case of the travelling plane wave when the function $U(x, y)$ is specified as $U(x, y)=\exp \left[\mathrm{i}\left( \pm h_{x} x \pm\right.\right.$ $\left.\left.h_{y} y\right)\right]$.

Furthermore, travelling waves are obtained when $U(\rho, \alpha)=c_{1} \mathrm{H}_{0}^{(2)}(\beta \rho)$. Then, the electromagnetic field configuration far from the symmetry axis becomes similar to the travelling wave and has the elliptical polarization. The polarization plane always is perpendicular to the slab surface.

\section{References}

[1] K. Remley, Analysis of the dielectric slab waveguide, in: WESCON/'93. Conference Record, 28-30 September 1993, pp. 646-651.

[2] D. Mugnai, Bessel beam throught a dielectric slab at oblique incidence: The case of total reflection, Opt. Commun. 207, 95-99 (2002).

[3] R.F. Harrington, Time-Harmonic Electromagnetic fields (Wiley, New York, 2001).

[4] E.J. Rothwell and M.J. Cloud, Electromagnetics (CRC Press, Florida, 2001).

[5] J.D. Jackson, Classical Electrodynamics (Wiley, New York, 1999).

Fig. 5. Electric field lines. $T E_{1}$ mode, $\varepsilon_{1}=10, \varepsilon_{2}=1, f=$ $1 \mathrm{GHz}$, symmetrical electric field case.

\title{
CILINDRINĖS BANGOS DIELEKTRINĖJE PLOKŠTELĖJE
}

\author{
V. Ivaška, V. Jonkus \\ Vilniaus universitetas, Vilnius, Lietuva
}

\section{Santrauka}

Gautos bendros dispersinès lygtys elektromagnetinèms bangoms, galinčioms sklisti begalinèje dielektrinejje plokštelejje. Elektromagnetinio lauko išraiškos gaunamos specialiai parinkus elektrinị arba magnetinį Herco potencialą, turintị tik statmeną plokštelei sandą. Gauti sąryšiai, siejantys bangos dažnį, medžiagos pa- rametrus ir bangos sklidimo konstantas. Šie saryšiai yra vienodi visoms elekromagnetinio lauko konfigūracijoms, kuriu pasiskirstymas plokštelèje aprašomas dvimate Helmholco lygtimi.

Parodyta, kad žinomi elektromagnetinio lauko pasiskirstymai, plokščios bangos, sklindančios ir stovinčios bangos seka kaip atskiras gautų išraiškų atvejis. 